(e-migrinter

e-Migrinter

$10 \mid 2013$

Genre et imbrication des rapports de domination dans les médias des minorités ethniques

\title{
Céline Barrère ; Claire Lévy-Vroelant, Hôtels meublés à Paris. Enquête sur une mémoire de l'immigration
}

Mélanie Pénicaud

\section{OpenEdition}

\section{Journals}

Édition électronique

URL : https://journals.openedition.org/e-migrinter/441

DOI : 10.4000/e-migrinter.441

ISSN : 1961-9685

Éditeur

UMR 7301 - Migrinter

Édition imprimée

Date de publication : 11 avril 2013

Pagination : 91-94

ISSN : 1961-9685

Référence électronique

Mélanie Pénicaud, « Céline Barrère ; Claire Lévy-Vroelant, Hôtels meublés à Paris. Enquête sur une mémoire de l'immigration », e-Migrinter [En ligne], 10 | 2013, mis en ligne le , consulté le 20 mai 2021.

URL : http://journals.openedition.org/e-migrinter/441 ; DOI : https://doi.org/10.4000/e-migrinter.441 
NOTE DE LECTURE

\section{Barrère, Céline ; Lévy-Vroelant, Claire (2012) Hôtels meublés à Paris. Enquête sur une mémoire de l'immigration, Paris, Créaphis, 300 p.}

Mélanie Pénicaud

$\mathbf{L}$ a démarche scientifique de l'ouvrage Hôtels meublés à Paris. Enquête sur une mémoire de l'immigration, menée par l'urbaniste Céline Barrère et la sociologue Claire Lévy-Vroelant, s'inscrit au croisement de plusieurs questionnements, controverses académiques et axes de recherche nouveaux en Sciences humaines et sociales. Bien que s'affirmant comme sous-tendue par la sociologie, la recherche engagée est résolument transdisciplinaire et les questionnements qu'elle porte de nature historique, sociologique, anthropologique, géographique, et littéraire. Tout en élevant leurs analyses en direction de préoccupations méthodologiques et disciplinaires, les deux chercheuses s'engouffrent dans un domaine jusqu'ici peu étudié par la sociologie en questionnant la mémoire des hôtels meublés parisiens. Si les problématiques mémorielles ont été jusqu'ici fortement travaillées par les historiens - dans le sillage des travaux de Pierre Nora et autour du concept de « lieu de mémoire ${ }^{1}$ notamment - et dans une moindre mesure par les anthropologues, la sociologie a encore peu investi ce domaine ${ }^{2}$. Plus encore, la mémoire de l'immigration au cœur de l'approche puisque ces hôtels demeurent encore aujourd'hui largement fréquentés par une population immigrée demeure encore un "champ à défricher» (p. 7).

\footnotetext{
${ }^{1}$ Nora Pierre (dir.), Les Lieux de mémoire, 1997, Paris : Gallimard, $4751 \mathrm{p}$.

${ }^{2}$ On ne peut cependant ne pas évoquer les travaux de Maurice Halbwachs, notamment: (1925) Les Cadres sociaux de la mémoire, Paris, Alcan, $211 \mathrm{p}$.
}

À travers une enquête qui cherche à restituer la mémoire de ces hôtels dans sa complexité et sa vivante réalité, Céline Barrère et Claire Lévy-Vroelant s'attachent à contourner la polémique contemporaine qui gravite autour de l'édification de lieux de mémoire monumentaux et de l'instrumentalisation de ces derniers dans la construction politique d'une identité nationale. C'est donc au regard de la banalité, de la marginalité et de l'anonymat des hôtels meublés, aujourd'hui menacés de disparition et d'oubli, que les chercheuses interrogent ces lieux de passage et leurs acteurs - les auteures parlent ici de «milieux de mémoire $»^{3}$ - comme autant de maillons de la chaine de la transmission d'une mémoire de l'immigration ${ }^{4}$. L'ouvrage prend donc une orientation diamétralement opposée de celle des travaux de Pierre Nora, pour qui les « lieux de mémoire » s'incarnent en tout objet matériel et immatériel (monuments, personnages, archives, évènements, symboles, devises, etc.) dès l'instant qu'il échappe à l'oubli en trouvant refuge dans la mémoire collective.

C'est alors, dans ces lieux paradoxaux où se côtoient circulation et installation, conversation nécessaire et silence salutaire,

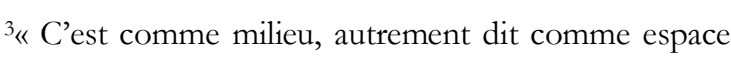
d'un groupe ayant en commun, de façon plus ou moins marquée, une histoire, des références, des valeurs et un mode de vie que nous avons saisi l'hôtel. » (p.10).

${ }^{4}$ Cette recherche a été impulsée par l'appel d'offre du Ministère de la Culture et de la Communication intitulé «Mémoire de l'immigration, vers un processus de patrimonialisation?».
} 
une mémoire de l'immigration en creux que révèlent les enquêtrices: une mémoire morcelée, parcellaire, lacunaire, mémoire d'un «entre-deux» pour ces espaces de l'exil, mémoire duelle aussi, englobant le temps long des hôteliers et celui, plus court, des clients de l'hôtel. Face à la fragmentation des récits de ceux qui ont fait l'expérience de ces hôtels loués au mois - et donc face à des questionnements méthodologiques de captation et de restitution de l'expérience - et dans le souci de rassembler un corpus le plus exhaustif possible se rapportant à cette mémoire, les auteures ont fait le pari d'intégrer une méthodologie originale à leur travail d'enquête de terrain en adjoignant à leur matériau un corpus littéraire. Aussi les «écritures des hôtels » sont-elles analysées comme autant de «mécanismes narratifs» participants à des processus de construction identitaires de lieux comme de mémoires. Les textes littéraires - d'auteurs maghrébins, africains ou haïtiens portant sur l'immigration et incluant la figure de l'hôtel sont prétextes à «recoupements» et «prolongations » du corpus issu du travail de terrain. Les chercheuses les ont sélectionnés selon des critères précis : qualitativement (genre littéraire, langue française, etc.) et, plus surprenant, quantitativement, à l'aide d'une base de données permettant de mettre en évidence l'occurrence de certains mots.

Une enquête sémantique et lexicale autour du terme "hôtel meublé », l'étude d'un corpus littéraire plus vaste encore, ainsi que la mobilisation des outils de la critique thématique et l'examen de la forme des expressions comme cadres de la mémoire viennent enrichir la méthode. Par là se mettent en place les mécanismes d'une approche décrite comme "subalterniste ${ }^{5}$ et écologiste (au sens de l'écologie urbaine), sensible et interactionniste» (p. 7).

${ }^{5}$ En prenant comme objet d'étude l'expression de mémoires contraintes par un «déficit de reconnaissance » (p. 33), les auteures se sont appuyées sur les travaux des Subaltern Studies.
L'invocation d'un corpus littéraire vient ainsi ouvrir la porte sur des expériences, incluant fantasmes, représentations, projections, identifications, affects, pulsions, etc., «donnant vie et corps à ce qui n'est pas saisissable par les archives » (p. 86).

Les auteures reconstruisent alors au fil des pages l'histoire des hôtels meublés : des rafles de juifs aux caches du FLN, de la succession des travailleurs ouvriers à une clientèle largement issue de l'immigration, en passant par les opérations de rénovation de l'action publique et les changements de clientèle dus aux locations de chambres, depuis les années 1990, par les services sociaux ou associations (ASE, Emmaüs...). Autant de situations qui bouleversent les rapports sociaux et le rythme des activités mémorielles. On retient pour exemple l'analyse des chercheuses quant au glissement de nombreux hôtels meublés vers le système des "hôtelleries dites sociales" (p. 193) : autant de «milieux» qui se muent en des espaces normés, surveillés, impersonnels, où les interactions sociales restent strictement professionnelles et desquels s'estompe toute «idée de groupe » (p. 186), et par là même de mémoire.

La seconde partie de l'ouvrage développe une sociologie de la mémoire de ces lieux habités, à travers l'étude des espaces de l'hôtel et de leurs fonctions sociales. L'hôtel se dévoile alors dans son entier comme un lieu de passage, un «seuil», à de multiples échelles et temporalités, en incarnant le lien entre l'ici et l'ailleurs, passé et présent, le dedans et le dehors, intime et extime. Par une étude détaillée des conditions d'élaboration des récits et des interactions en place doublée d'une lecture fine du corpus littéraire, les auteures dépeignent l'hôtel comme marqué par des rapports de domination - internes et externes - et des situations de surveillance, mais aussi de «care, de médiation et 
d'endiguement» (p. 147) opérées par les hôteliers : autant d'éléments qui participent des représentations collectives et structurent la mémoire du «milieu » et les conditions de son émergence. Ici, l'analyse thématique permet de mettre en lumière la récurrence des thèmes de la mort, de la dette, des travaux, etc., qui nourrissent la mémoire de ces lieux naviguant entre passé et présent. Celle-ci se structure autour de normes, de codes implicites et de jeux sociaux (un certain art de la conversation par exemple) qui encadrent les coprésences en place : ces dispositions sont mises au jour par les auteures après de nombreux questionnements sur les notions d'identité individuelle, d'identité collective, de groupe, de communauté, de famille, etc.

Lieu de ruptures et de résiliences, de ceux qui mettent la mémoire au défi, l'hôtel meublé apparait alors dans la troisième partie de l'ouvrage comme un milieu de restructuration et de réappropriation d'une mémoire de l'immigration parcellaire. Les chercheuses relèvent trois thématiques principales au cœur de cette mémoire: en premier lieu, l'histoire et la géographie (l'hôtel comme «milieu» ne saurait être détaché d'un contexte spatio-temporel) dont elles étudient les représentations. Ensuite les langues qui, au-delà des complexes induits par une maitrise malhabile, deviennent les objets d'un "patchwork linguistique » (p. 246) et de jeux de langage. Elles analysent en dernier lieu l'expression des cultures propres et du mélange de cellesci, des constructions culturelles et identitaires induites: transferts, mises en relations, hybridations, toujours à saisir dans un contexte social, politique, etc. Autant de «bricolages identitaires » à l'œuvre dans le microcosme de l'hôtel, fondés sur des rapports de forces élaborés en son sein parfois émancipés de ceux habituellement institués - qui surgissent des discours et des récits dont les formes anecdotiques ne sont pas pour autant écartées de la plume des chercheuses. Apparaissent ainsi des formes de sociabilité et des figures de l'altérité impensées qui, au delà d'enrichir les études sur la mémoire de l'immigration, ajoutent à la compréhension des expériences migratoires. L'hôtel, dans son corps social et physique, se révèle ainsi par excellence le lieu de la fragmentation, de l'inachèvement et du provisoire, posant de manière aigüe les questions de l'expression et de la transmission mémorielles. Il devient alors le lieu où se dessine une "identité de la relation ${ }^{6}$ marquée des sceaux du don et de la dette, dans un milieu et à un temps de l'expérience migratoire propice à la réécriture de soi "après les mouvements d'effraction et de gommage produits par la colonisation comme par l'immigration» (p. 251).

C'est ainsi que l'hôtel, en tant que «milieu de mémoire de l'immigration» (p. 229), est de ces «espaces limites» (p. 244) qui viennent mettre en lumière les impasses du récit de soi et de la restitution des expériences migratoires. Conscient de cette complexité, l'ouvrage dans son ensemble, par la multiplication des supports méthodologiques et par l'ensemble des choix visuels et explicatifs qui ont été faits, parvient à contourner ces obstacles venant jusqu'ici entraver la reconnaissance d'une mémoire de l'immigration en des espaces marginaux et marqués par le silence, mais dans lesquels, pourtant, la parole est reine et les questions de transmission souvent centrales. Le travail de Céline Barrère et Claire Lévy-Vroelant parvient à retranscrire, situer et transmettre à son tour au lecteur la fragmentation et l'épaisseur d'une mémoire de l'immigration, les possibles de sa transmission et les variations des formes de «socialisations marginales" (p. 255) à l'œuvre dans ces milieux de passage. Par ailleurs, la littérature ne serait-elle pas finalement le seul vecteur fidèle possible de la mémoire de ces hôtels meublés qui semble insaisissable par toute entreprise de

${ }^{6}$ Expression que les chercheuses utilisent en référence à la Poétique de la relation d'Édouard Glissant. 
patrimonialisation? Suggérant de nombreuses voies encore inexplorées, l'ouvrage lève le voile sur les hôtels meublés, ces impensés de la mémoire de l'immigration.
Mélanie Pénicaud Doctorante en anthropologie Migrinter - UMR 7301 CNRS/Université de Poitiers melaniepenicaud@gmail.com 